\title{
Statistical Atlases of Bone Anatomy: Construction, Iterative Improvement and Validation
}

\author{
Gouthami Chintalapani ${ }^{1}$, Lotta M. Ellingsen ${ }^{2}$, Ofri Sadowsky ${ }^{1}$, \\ Jerry L. Prince ${ }^{2}$, and Russell H. Taylor ${ }^{1}$ \\ Johns Hopkins University, Baltimore, USA \\ greddy@cs . jhu . edu
}

\begin{abstract}
We present an iterative bootstrapping framework to create and analyze statistical atlases of bony anatomy such as the human pelvis from a large collection of CT data sets. We create an initial tetrahedral mesh representation of the target anatomy and use deformable intensitybased registration to create an initial atlas. This atlas is used as prior information to assist in deformable registration/segmentation of our subject image data sets, and the process is iterated several times to remove any bias from the initial choice of template subject and to improve the stability and consistency of mean shape and variational modes. We also present a framework to validate the statistical models. Using this method, we have created a statistical atlas of full pelvis anatomy with 110 healthy patient CT scans. Our analysis shows that any given pelvis shape can be approximated up to an average accuracy of $1.5036 \mathrm{~mm}$ using the first 15 principal modes of variation. Although a particular intensity-based deformable registration algorithm was used to produce these results, we believe that the basic method may be adapted readily for use with any registration method with broadly similar characteristics.
\end{abstract}

\section{Introduction}

Statistical modeling and analysis of anatomical shape is an active subject of medical imaging research. Uses include image segmentation, analysis of anatomical variations within populations, identification of pathological anomalies, etc. Statistical characterization using principal component analysis (PCA) and point distribution models is presented in [1. Following Cootes et al., a number of authors (e.g., [2, 3], 4], 5]) have applied similar methods to construct statistical atlases of bony anatomy from CT scans of multiple individuals. The basic method used is to identify landmark points, establish point-based correspondences between subjects, and then perform statistical analysis to study shape variations. Typically, subject anatomical shapes are represented as surface meshes 4 or volumetric tetrahedral meshes [2, [5], 3]. Often, a template mesh representing an anatomical structure is created, deformably registered to each subject, and mesh vertex points are used as the corresponding landmark points. Wu et al. created a statistical model using surface triangular meshes and a non-rigid point matching 
algorithm to register different shapes. Our atlas approach, originated by Yao et al. [2] and subsequently also adopted by others [5] [3], represents the shape using tetrahedral meshes and registers the datasets using a grayscale deformable registration method. Bone density information is incorporated into the model using polynomials. Some drawbacks of these methods are that a bias is introduced in selecting a template shape and the sampling points on the surface are over determined when a deformable registration method is used. Bookstein et al. proposed an iterative ridge curve based algorithm to register each curve/shape to the average shape 6. However, this approach poses difficulties when applied to volumetric data. Chui et al. presented an iterative process where multiple sample point sets are non rigidly deformed to the emerging mean shape [7]. Chui's method requires that each subject be separately segmented to identify the points. Although he suggests that the method can be useful in atlas construction, he does not address evolution of statistical modes. Rueckert et al. [8] constructed an intensity atlas by using deformable 3D-3D intensity image-toimage based registration to approximate the deformations between subjects and a template using B-spline polynomials, and then constructed a statistical atlas to the B-spline control points.

This paper proposes a systematic method for construction and iterative bootstrapping of a statistical mesh atlas from multiple CT data sets. Our approach most closely follows Yao's. It resembles Rueckert's by using image-to-image intensity-based registration to determine an initial set of deformations, but differs by applying them to our mesh data structure, in the use of PCA on the mesh to produce statistical modes, and in the use of 3D-3D atlas-to-image intensitybased registration in iterative bootstrapping. Although the method is potentially useful with any deformable mesh registration algorithm capable of incorporating prior statistical information, we demonstrate it with a new method that incorporates whatever prior information is available. We present validation results on a full pelvis atlas created from 110 datasets using this iterative process. We present the algorithm and results below.

\section{Method}

This section describes the iterative process of creating the statistical atlas. The statistical atlas consists of a tetrahedral mesh representing the average shape, Bernstein polynomials representing the CT intensities and the variational modes representing shape variations. A typical cross sectional view of the tetrahedral mesh skeleton is shown in Figure 1]. Although our current atlas includes bone density parameters, for the purpose of this paper we are restricting ourselves to shape parameters. Our atlas construction method relies on the use of a deformable registration method developed by Ellingsen et. al [9] that has been modified to use prior statistical knowledge from our atlas in registering the volumetric datasets. Several authors (e.g., [2, [10, 11]), have proposed similar registration methods. Generally, these methods include the following steps: 1) similarity 


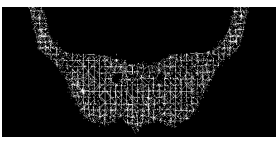

(a)

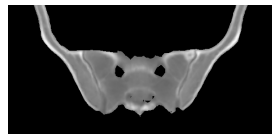

(b)

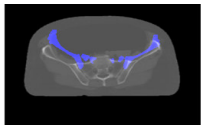

(c)

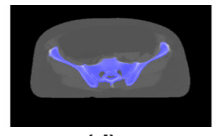

(d)

Fig. 1. (a) A cross-sectional view of tetrahedral mesh skeleton (b) mesh fitted with density polynomials (c) a CT slice of a typical subject with voxelized tetrahedral mesh (in blue) overlaid before registration (d) after registration

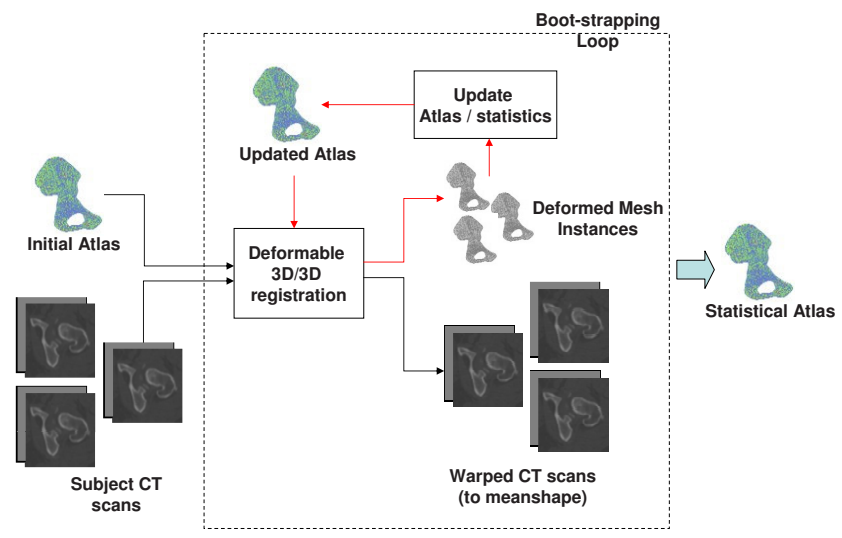

Fig. 2. Statistical atlas creation process using iterative bootstrapping technique

registration, 2) statistical mode matching, and 3) local deformation. In our current implementation, the gray scale deformable registration algorithm uses a continuously varying weighting parameter to smoothly vary from mode matching to purely image-driven local deformation, while preserving correct mesh topology.

A flowchart describing the iterative process is shown in Figure 2 and the algorithmic steps are shown below:

1. Select a template CT dataset $C_{\text {master }}$ and manually label the voxels of the anatomy of interest, resulting in $C_{\text {master }}^{\text {segmed }}$. Create a tetrahedral mesh $M_{\text {master from }} C_{\text {master }}^{\text {segmed }}$. We have used the meshing application developed by 12 .

2. Select $n_{\text {seed }}$ CT datasets from the population, say $C_{i}$, where $i=1,2,3, . . n_{\text {seed }}$. Deformably register each dataset to the template $C_{\text {master }}$ using a $3 \mathrm{D}$ grayscale deformable registration. The output of this step would be a deformation field $\operatorname{Dmap}_{i}$ and a warped subject CT, $C_{i}^{\text {warped }}$.

3. For each CT subject, create a mesh instance, $M_{i}$ by interpolating the deformation field $D_{i}$ at each vertex of the master mesh $M_{\text {master }}$. 
4. Do a rigid registration between all the meshes and perform principal component analysis on the registered mesh instances. This results in meanshape $M_{0}$ and shape variational modes $D_{k}$, refered to as Atlas $j$ where $j=0$. Any given shape can be expressed as a linear combination of the anatomical modes of variation

$$
S=M_{0}+\Sigma_{k=1}^{n} \lambda_{k} D_{k}
$$

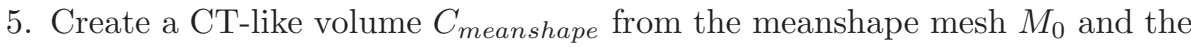
mean density polynomial.

6. For each CT subject $C_{i}$, deformably register $C_{i}$ to $C_{\text {meanshape. This reg- }}$ istration method uses prior knowledge (meanshape $M_{0}$ and modes $D_{i}$ ) to constrain the deformation process and to increase the registration accuracy. The resulting deformed mesh instance is $M_{i}$

7. Compute a new statistical model using principal component analysis on the mesh instances. The result of this step is a meanshape $M_{0}^{j}$ and modes $D_{i}^{j}$, refered to as Atlas $_{j+1}$

8. Compare the two models, $\Delta=$ Compare $\left(\right.$ Atlas $_{j}$, Atlas $\left._{j+1}\right)$. Various tools for comparing any two given models are presented in the following section. If $\Delta>\epsilon$, then $j=j+1$ and iterate steps 7 through 10. Stop otherwise.

With this bootstrapping process, the vertex correspondences are stabilized and the residual variance after principal component analysis is reduced after each iteration. Moreover, this iterative procedure removes the bias introduced in selecting the template subject and removes the artifacts introduced by the registration algorithms, thereby stabilizing the anatomical modes of variation. Our atlas creation process is modular and robust and we believe that the underlying concept can be readily adapted for use with any similar deformable registration method.

\section{Results}

We have created a statistical model of full pelvis anatomy from $110 \mathrm{CT}$ scans of healthy patients using this new iterative method. The tetrahedral mesh model consists of 26875 vertices, 105767 tetrahedra, and 25026 outer surface triangles. This mesh was created from a $512 \times 512 \times 256 \mathrm{CT}$ volume with a voxel size of 0.9375 $\mathrm{cm}^{3}$. Results from four iterations of this boot-strapping method are presented. The zeroth iteration corresponds to basic atlas creation by registering all the subjects to a template subject without any prior knowledge. In later iterations, we use the statistical model from the previous iteration as a template. Figure 3 shows surface rendered mean shape and the first three modes of pelvis anatomy from the final iteration.

For validation purpose, we have randomly selected 20 datasets from our CT population and excluded all these 20 datasets from the atlas creation. The goal is to estimate these 20 left out subjects from atlases at different stages. Given a leftout shape instance, $S^{\text {true }}$, align this shape instance with the mean shape using similarity transformation. And then the shape can be estimated with deformable 


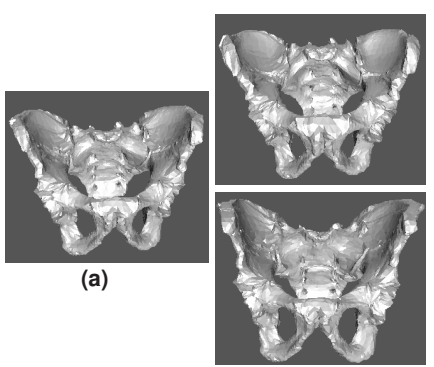

(b)

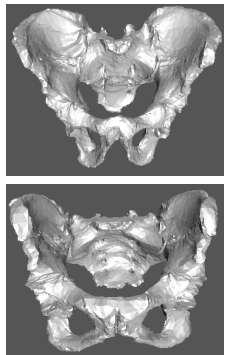

(c)

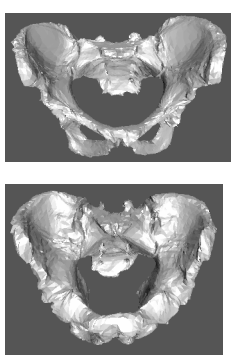

(d)

Fig. 3. Surface renderings of mean shape and first three principal modes. (a) mean shape; (b) top: mean $+3 \sigma 1$ mode1, bottom: mean- $3 \sigma 1$ mode1; (c)top: mean $+3 \sigma 2$ mode 2 , bottom: mean- $3 \sigma 2$ mode 2 ; (d) top: mean $+3 \sigma 3$ mode 3 , bottom: mean- $3 \sigma 3$ mode 3 ;

mode matching step as follows: Compute the mode weights using the mean shape (overline $M$ ) and the modes of variation $(Y)$

$$
\lambda=Y^{\prime} *\left(S^{\text {true }}-\bar{M}\right)
$$

Use the dominant eigen modes to estimate the given shape

$$
S^{e s t}=\bar{M}+\sum_{i=1}^{n} \lambda_{i} Y_{i}
$$

After estimating a given shape with the atlas, we define two types of metrics to measure the error between the estimated shape and the true shape: 1) vertex to vertex correspondence error assuming that the graphs of the meshes are similar and 2) surface to surface distance computed by measuring distances from the vertices of the model instance to the closest points on the subject surface [13. We have performed four iterations of the boot-strapping algorithm and the residual error plots are shown below. The residual error as estimated from the leaveout validation tests in each iteration of the bootstrapping process is shown in Figure 4. For each iteration, we have created an atlas using 90 datasets and
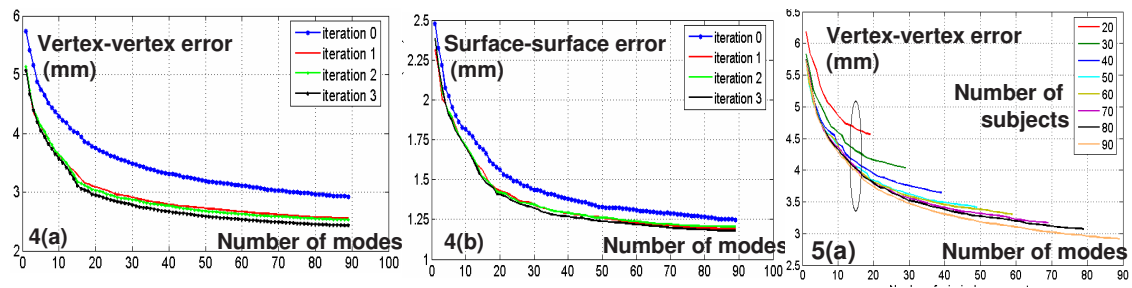

Fig. 4. Residual error plots from leave-out validation tests as a function of number of principal modes: (a) vertex to vertex correspondence error; (b) surface to surface distance; (c) Vertex to vertex correspondence error as a function of number of principal components used shown for different numbers of CT subjects used to form the atlas; 
performed leave-out validation experiments using the remaining 20 datasets. Figure 4 shows that after first two iterations, the process more or less converged. There is a significant reduction in the vertex to vertex correspondence metric from iteration 0 to iteration 1 indicating that the vertex correspondences have improved. This process seems to have converged after iteration 2. A similar trend can be seen with surface to surface distance error metric. We have used our surface to surface distance metric to select the number of principal components to be used in the later experiments. Even though large number of eigen modes result in lower residual errors, it is computationally expensive to use a large set of principal modes. Typically, a threshold on the residual errors is used to determine the number of eigen modes. Here, we set the surface distance threshold to be approximately $1.5 \mathrm{~mm}$ and hence selected the first 15 shape modes in later experiments.

In order to analyze the size of the population and the number of principal modes needed to extract stable statistics, we performed the following experiment. We randomly selected $n$ meshes, where $n=20,30,40, . .80$ and created statistical models using these datasets. This process was repeated 20 times for each value of $n$. Figure 4(c) shows the average residual vertex correspondence error in the

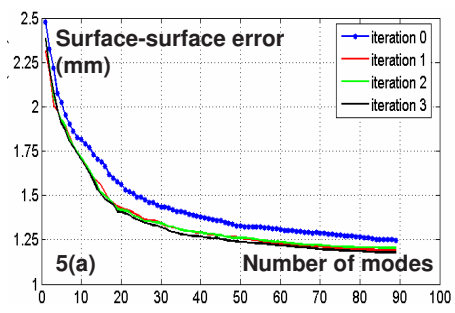

\begin{tabular}{|c|c|c|c|c|}
\hline & Iter 0-1 & Iter 1-2 & $\begin{array}{c}\text { Iter 1- } \\
3\end{array}$ & Iter 2-3 \\
\hline Meanshape & 4.4778 & 0.138 & 0.127 & 0.126 \\
\hline $\begin{array}{c}\text { Meanshape + } \\
400^{*} \text { Mode 1 }\end{array}$ & 4.2293 & 0.5759 & 0.5872 & 0.4938 \\
\hline $\begin{array}{c}\text { Meanshape + } \\
400^{*} \text { Mode2 }\end{array}$ & 4.3908 & 0.7412 & 0.834 & 0.8259 \\
\hline $\begin{array}{c}\text { Meanshape + } \\
400^{*} \text { Mode3 }\end{array}$ & 5.2305 & 0.6320 & 0.6521 & 0.4688 \\
\hline
\end{tabular}

Fig. 5. (a) Residual surface to surface distance error from using iteration 3 as ground truth; (b) Surface to surface distance metric between mean shapes and modes from iterations $0-3$

leave-out tests for different atlases as a function of number of modes used for various atlas population sizes. This graph shows that around 40 to 50 datasets are sufficient to capture the shape variations of a healthy pelvis anatomy using 15 modes. Adding more instances to this database results in a very small improvement, less than $0.1 \mathrm{~mm}$, in terms of residual errors.

We analyzed the convergence in terms of the stability of the atlas. This analysis is shown in Table 1. The surface to surface distance between mean shapes from iterations 2 and 3 is around $0.126 \mathrm{~mm}$ and the volume overlap between the mean shape CT volumes is around $97 \%$. Similarly, the average surface to surface distance betwen the shape modes for iterations 1,2 , and 3 is around $0.6 \mathrm{~mm}$. After iteration 2, the mean shape and modes did not vary much. These values indicate that the atlas has become stable and the bootstrapping process has converged. As one of the reviewers mentioned, it could so happen that the process has converged to a consistent sub-optimal solution rather than the optimal 
solution. The intuition behind the iterative process is that after every iteration, residual errors are decreased by deforming/stretching the mesh vertices beyond the PCA mode matching step. To verify the convergence against ground truth, we selected mesh instances from the final iteration as our ground truth shapes. and performed leave-out validation analysis. The bootstrapping process has converged in iteration 3 as shown in Figure 5(b). However, we have measured the closest point distance between a few hand segmented meshes and the corresponding atlas instances from the final iteration. The average error is around 2.0153 $\mathrm{mm}$. This number is roughly comparable to the $1.5036 \mathrm{~mm}$ accuracy achieved from using final iteration as ground truth. However, it is difficult to interpret these numbers in the absence of a firm consensus segmentation since manual segmentations are subject to random errors. We plan to do a thorough systematic evaluation of the algorithm against a consensus from multiple independent segmentations.

\section{Conclusions and Future Work}

This paper has presented an iterative bootstrapping technique to create statistical atlases of bony anatomy from collections of CT data sets, along with various error metrics to evaluate these atlases. Advantages of our approach include: 1) very minimal initial segmentation is required (once to create an initial mesh) and may be done manually or semi-automatically; 2) point correspondences are established automatically through intensity-based registration, avoiding landmark selection; 3) atlas bias/uncertainty is minimized through iterative refinement of an initial atlas; 4) the method requires little or no explicit prior anatomical information, although such information may be added in a separate annotation phase; and 5) the atlases produced are useful as prior information for assisting 3D/3D and 2D/3D (e.g., 5]) registration, as well as assisting in tomographic reconstruction from incomplete data [5].

Although we used a particular intensity-based registration method 9] to create this atlas, the technique can be adapted readily for use with other registration algorithms, although this must be demonstrated in actual experiments. Our method is modular and robust, thereby enabling us to build large population atlases fairly easily. As part of the validation framework, we have developed various tools and strategies to compare and analyze any given number of statistical models. In this paper, we have focused on shape statistics only and we are currently using mean density for our applications. An immediate extension would be to incorporate density statistics in to our atlas construction pipeline and combine the shape and density statistics to give us a better atlas.

We have demonstrated this approach by creating an atlas of the human male pelvis from 110 subjects. Our population analysis shows that up to 40-50 subjects are required to capture inherent shape variations of pelvic bone anatomy, although additional subjects further improve the results somewhat. The leaveout validation experiments indicate an accuracy of $1.5036 \mathrm{~mm}$ in approximating any given pelvis shape with the first 15 eigen modes of the 90 dataset atlas. Our 
next experiment will be to validate the bootstrapping process with a consensus multiple independent segmentations. In related ongoing work, we will shortly apply our boot-strapping approach to create male and female femur atlases.

\section{Acknowledgments}

We would like to thank Dr. Ted Deweese and Dr. Lee Myers for their assistance in providing us with the data. This work is supported by NSF ERC Grant EEC9731478 and NIH/NBIB Grant R21-EB003616. We would also like to thank Pauline Pelletier for helping us with data processing.

\section{References}

1. Cootes, T., Taylor, C., Cooper, D., Graham, J.: Active shape models - their training and application. Computer Vision Image Understanding 61(1), 38-59 (1995)

2. Yao, J., Taylor, R.H.: Non-rigid registration and correspondence finding in medical image analysis using multiple-layer flexible mesh template matching. IJPRAI 17(7), 1145-1165 (2003)

3. Querol, L., Buchler, P., Rueckert, D., Nolte, L.P., Ballester, M.: Statistical finite element model for bone shape and biomechanical properties. In: Larsen, R., Nielsen, M., Sporring, J. (eds.) MICCAI 2006. LNCS, vol. 4190, pp. 405-411. Springer, Heidelberg (2006)

4. Wu, C., Murtha, P.E., Mor, A.B., Jaramaz, B.: A two-level method for building a statistical shape atlas. In: CAOS (2005)

5. Sadowsky, O., Ramamurthi, K., Ellingsen, L., Chintalapani, G., Prince, J., Taylor, R.: Atlas-assisted tomography: registration of a deformable atlas to compensate for limited-angle cone-beam trajectory. In: IEEE International Symposium for Biomedical Imaging pp. 1244-1247 (2006)

6. Cutting, C.B., Bookstein, F.L., Haddad, B., Dean, D., Kim, D.: Spline-based approach for averaging three-dimensional curves and surfaces. Mathematical methods in medical imaging II, SPIE 2035, 29-44 (1993)

7. Chui, H., Zhang, J., Rangarajan, A.: Unsupervised learning of an atlas from unlabeled point-sets. IEEE Trans. Pattern Analysis and Machine Intelligence 26(2), 160-173 (2004)

8. Rueckert, D., Frangi, A., Schnabel, J.: Automatic construction of 3d statistical deformation models using non-rigid registration. In: Medical Image Computing and Computer Assisted Intervention, pp. 77-84 (2001)

9. Ellingsen, L., Prince, J.: Deformable registration of ct pelvis images using mjolnir. In: IEEE 7th Nordic Signal Processing Symposium (NORSIG) (2006)

10. Cootes, T., Beeston, C., Edwards, G., Taylor, C.: A unified framework for atlas matching using active appearance models. In: IPMI, pp. 322-333 (1999)

11. Shen, D., Davatzikos, C.: Adaptive-focus statistical shape model for segmentation of 3d mr structures. In: Delp, S.L., DiGoia, A.M., Jaramaz, B. (eds.) MICCAI 2000. LNCS, vol. 1935, pp. 206-215. Springer, Heidelberg (2000)

12. Mohamed, A., Davatzikos, C.: An approach to 3d finite element mesh generation from segmented medical images. In: IEEE International Symposium on Biomedical Imaging (ISBI) (2004)

13. Besl, P., McKay, N.: A method for registration of 3-d shapes. IEEE Trans. on Pattern Analysis and Machine Intelligence 14(2), 239-256 (1992) 